\title{
Role of clip markers placed by endoscopic ultrasonography in contouring gross tumor volume for thoracic esophageal squamous cell carcinoma: one prospective study
}

\author{
Yong Guan ${ }^{1 \#}$, Jing Wang ${ }^{1 \#}$, Fuliang $\mathrm{Cao}^{2}$, Xi Chen ${ }^{1}$, Yuwen Wang ${ }^{1}$, Shengpeng Jiang ${ }^{1}$, Daguang Zhang ${ }^{1}$, \\ Wencheng Zhang ${ }^{1}$, Zhoubo Guo ${ }^{1}$, Ping Wang', Qingsong Pang ${ }^{1}$ \\ ${ }^{1}$ Department of Radiotherapy, ${ }^{2}$ Department of Endoscopy, Tianjin Medical University Cancer Institute and Hospital, National Clinical Research \\ Center for Cancer, Key Laboratory of Cancer Preventing and Therapy, Tianjin's Clinical Research Center for Cancer, Tianjin, China \\ Contributions: (I) Conception and design: Y Guan, J Wang, Q Pang; (II) Administrative support: Q Pang, P Wang; (III) Provision of study materials: \\ F Cao, S Jiang, D Zhang; (IV) Collection and assembly of data: Z Guo, X Chen, Y Wang; (V) Data analysis and interpretation: Y Guan, W Zhang; (VI) \\ Manuscript writing: All authors; (VII) Final approval of manuscript: All authors. \\ \#These authors contributed equally to this work. \\ Correspondence to: Qingsong Pang. Tianjin Medical University Cancer Institute and Hospital, Huanhuxi Road, Hexi District, Tianjin, China. \\ Email: pangqingsong2013@163.com.
}

Background: We aimed to analyze the value of metal clip markers guided and placed by endoscopic ultrasonography (EUS) in the delineation of gross tumor volume (GTV) for thoracic esophageal squamous cell carcinoma.

Methods: From September 2016 to September 2018, patients with thoracic esophageal squamous cell carcinoma in Tianjin Medical University Cancer Institute and Hospital were recruited in the prospective trial, NCT02959385. They underwent titanium clips placement on tumor superior and inferior boundaries under EUS by a single expert endosonographer before radiotherapy computed tomography (CT) simulation. According to the clip markers, the reference GTVs were contoured by one experienced radiation oncologist. With the help of the Eclipse treatment planning system, clip markers on CT were concealed. Afterward, two other radiation oncologists with expertise in esophageal cancer delineated GTVs, defined as conventional GTVs, based on endoscopy and barium radiography findings. The two GTVs were compared and analyzed. Subgroup analysis was conducted in different $\mathrm{T}$ stage [early $(\mathrm{T} 1+\mathrm{T} 2)$ vs. advanced $(\mathrm{T} 3+\mathrm{T} 4)]$, focus location (upper $v s$. middle $v s$. lower segment), and tumor length $(<5 v s .>5 \mathrm{~cm})$ groups.

Results: The trial recruited 55 patients with 60 thoracic esophageal cancer foci. A total of 111 titanium clips were guided and implanted by EUS. Before CT simulation, two titanium clips at two foci fell off. After the procedure, no case of intolerable esophageal pain, hemorrhage, or perforation occurred. Compared to reference GTVs', discrepancies of conventional GTVs' superior borders were $0.91 \pm 0.82 \mathrm{~cm}(\mathrm{P}<0.001)$, while differences of inferior borders were $0.74 \pm 0.63 \mathrm{~cm}(\mathrm{P}<0.001)$. On the contrary, conventional GTVs' lengths were not significantly different from reference GTVs' with discrepancies $0.08 \pm 1.30 \mathrm{~cm}(\mathrm{P}=0.64)$. Regardless of T stage, tumor location, and tumor length, conventional GTVs' superior and inferior borders were significantly different from reference GTVs', while GTVs' lengths differed insignificantly.

Conclusions: This study confirmed that EUS-placed titanium clips could correct contouring of GTVs in thoracic esophageal cancer in different $\mathrm{T}$ stages, tumor locations, and lengths.

Keywords: Esophageal cancer; endoscopic ultrasonography (EUS); titanium clip; radiotherapy; gross tumor volume (GTV)

Submitted Feb 15, 2020. Accepted for publication Aug 19, 2020.

doi: 10.21037/atm-20-4030

View this article at: http://dx.doi.org/10.21037/atm-20-4030 


\section{Introduction}

It was estimated that 572,034 new cases with esophageal cancer occurred globally in 2018, while 508,585 patients died of the disease, ranking the sixth most fatal malignancy (1). Therefore, there is an urgent need to treat and cure esophageal cancer. Endoscopic submucosal resection for early stage esophageal carcinoma, minimally invasive esophagectomy, molecular target therapy and immunotherapy have contributed to recent advancement in treatment of esophageal cancer. Among them, radiotherapy is one of the mainstay treatment modalities, both in the settings of neoadjuvant chemo-radiotherapy for operable patients and definitive chemoradiation for inoperable patients $(2,3)$.

Since gross tumor volume (GTV) could influence the scope of the clinical target volume (CTV) and planning target volume (PTV) significantly, it is very imperative to contour GTV accurately for radiotherapy treatment planning and delivery, especially in the era of precision therapy. However, the delineation of GTV in radiotherapy for esophageal cancer varied across different researches. Although radiotherapy planning must be based upon radiation dose calculation on $\mathrm{CT}$ images, $\mathrm{CT}$ alone cannot facilitate accurate contouring of GTV for thoracic esophageal cancer adequately, given the fact that consistency between lesions' length measured by CT and measured after cancer surgery was only $32 \%$ (4). As a result, in routine clinical practice, we must refer to data of endoscopy and upper digestive tract radiography as well. However, due to the difference in patients' body positions and esophageal stretch during the process of these examinations, borders of GTV still could not be determined precisely on CT images. Subsequently, some researches utilized esophageal magnetic resonance imaging (MRI) or positron emission tomography-computed tomography (PET-CT) to assist delineating GTV more precisely $(5,6)$. Nevertheless, these novel methods also introduced new problems, such as complicated image registration, inconsistent interpretation of MRI or PET parameters, and high cost (5,7-10). For example, so far, the optimal PET modality for GTV estimation remains controversial (11).

Endoscopic ultrasonography (EUS) has been used in the diagnosis and staging of esophageal cancer for three decades. In comparison with conventional endoscopy, it could discover not only the superficial focus but also the indepth tumor, facilitating detecting borders of esophageal cancer in every layer. It was reported that EUS had excellent accuracy in staging superficial esophageal carcinoma (12).
In 2000, Riepl and colleagues reported the successful use of endoscopic clips in marking gastrointestinal tumors (13). In theory, EUS could place clip markers at tumor borders more precisely than conventional endoscopy. It was reported that, the accuracy of tumor marking could be enhanced by EUS because profound tumors could also be detected (14). Marking tumor borders with radiopaque titanium clips under EUS could avoid the shortcomings of other methods, such as non-visualization on simulation CT images, inaccurate determination of tumor borders, and prohibitive cost.

Consequently, we performed this prospective clinical trial, whose secondary endpoint was GTVs' accuracy. We aim to determine the impact of EUS-placed titanium clips on GTV contouring in radiotherapy for thoracic esophageal squamous cell carcinoma. Although some previous similar studies $(15,16)$ focused on efficacy of EUS in T staging of esophageal cancer, this is the first prospective research on comparison between esophageal GTVs with or without the help of EUS-guided clips in the same CT image set.

We present the following article in accordance with the STROBE reporting checklist (available at http://dx.doi. org/10.21037/atm-20-4030).

\section{Methods}

\section{Patients}

From September 2016 to September 2018, 55 patients with thoracic esophageal squamous cell carcinoma in Tianjin Medical University Cancer Institute and Hospital were recruited in the prospective trial, NCT02959385, whose secondary endpoints included determination of the role of metal clip markers in the delineation of esophageal cancer GTV. The recruitment criteria included the following: pathologically proven thoracic esophageal cancer patients, ranging from 18 to 70 years old, with $\mathrm{WHO}$ performance status $0-1$, clinically identified as stage $\mathrm{T} 1-4 \mathrm{~N}+\mathrm{M} 0$ according to UICC (International Union Against Cancer) TNM Classification of Malignant Tumors, 7th edition, without previous antitumor therapy, and life expectancy $>6$ months. The patients' blood examination should meet the following criteria: white blood cell count $\geq 4.0 \times 10^{9} / \mathrm{L}$, absolute neutrophil count (ANC) $\geq 1.5 \times 10^{9} / \mathrm{L}$, thrombocyte count $\geq 1.0 \times 10^{11} / \mathrm{L}$, hemoglobin $\geq 90 \mathrm{~g} / \mathrm{L}$ and normal liver and kidney functions. The exclusion criteria included the following: patients who have already received antitumor therapy, including chemotherapy, radiotherapy or surgery; patients with complicated hemorrhage; patients who are 

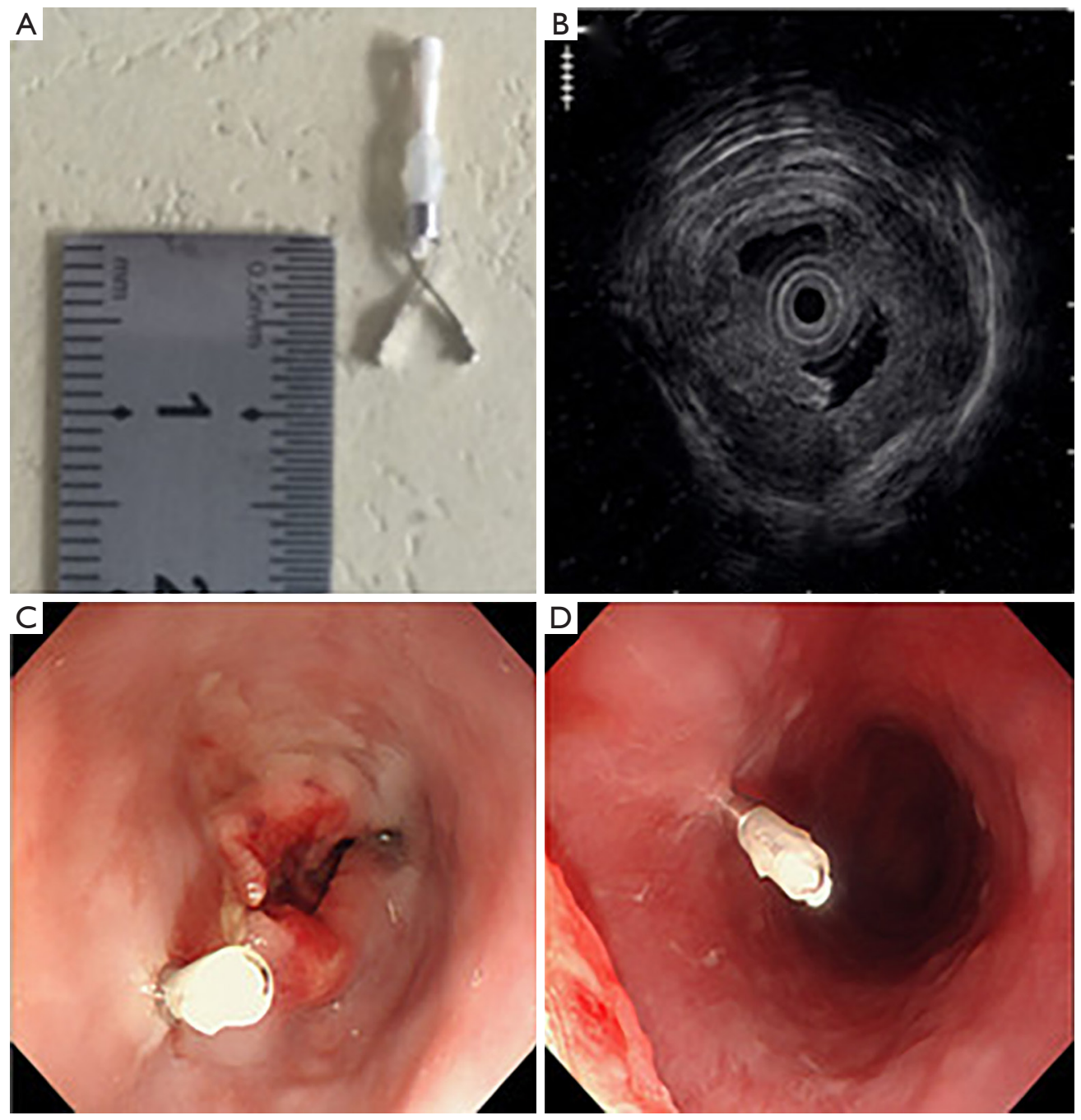

Figure 1 Titanium clip and placement of clips at borders of esophageal cancer under EUS. (A) Size of a titanium clip; (B) EUS view of an esophageal cancer focus; (C) superior border of this focus marked by a titanium clip; (D) inferior border of this focus marked by a titanium clip. EUS, endoscopic ultrasonography.

not suitable for surgery; pregnant or lactating women; patients who disagree with the informed consent as a result of psychological, family or social factors; patients with CTCAE(Common Terminology Criteria Adverse Events Version 4.0) grade $\geq 2$ peripheral neuropathy; patients who have ever had malignant tumors other than esophageal cancer; patients with a history of diabetes for $>10$ years with unsatisfactory control of blood glucose; patients with serious heart, lung, liver or kidney dysfunction, hematopathy, immune system disease or cachexia who therefore cannot tolerate chemotherapy or surgery.

All procedures performed in this study involving human participants were in accordance with the Declaration of
Helsinki (as revised in 2013). The study was approved by institutional ethics committee of Tianjin Medical University Cancer Institute and Hospital (No. E2016103A) and informed consent was taken from all the patients.

\section{EUS-guided clip marker placement}

All the patients underwent EUS under conscious sedation before radiotherapy CT simulation.

The Olympus EvisLucera CLV-260 SL EUS (Olympus Medical Systems Corp., Tokyo, Japan) was used to assess the $\mathrm{T}$ stage of tumor and place titanium clips at tumor borders (Figure 1). Titanium clips (Olympus Medical Systems Corp., 

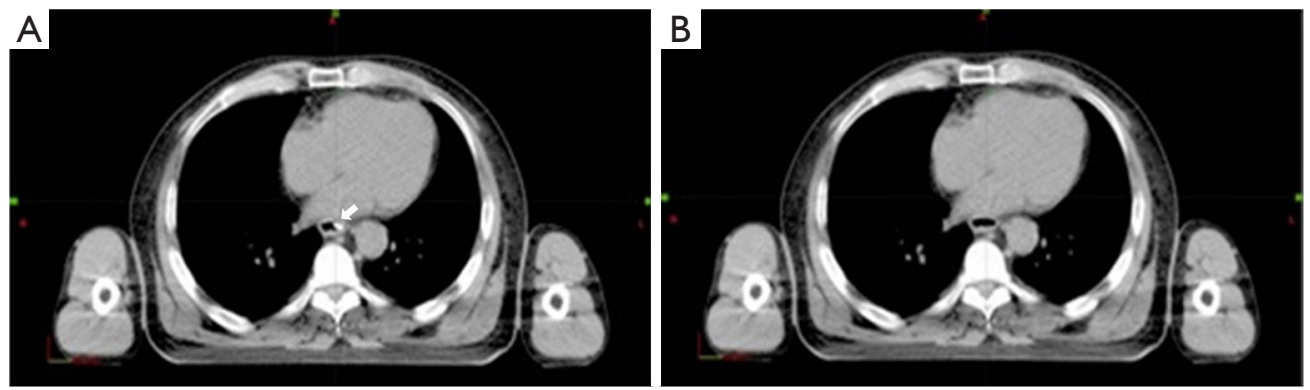

Figure 2 CT images of one patient with lower thoracic esophageal cancer, demonstrating difference between images before (A) and after (B) concealment of a titanium clip, as indicated by the white arrow. CT, computed tomography.

Table 1 Patients' and foci characteristics

\begin{tabular}{|c|c|c|}
\hline Characteristics & Number & Percentage \\
\hline \multicolumn{3}{|l|}{ Gender } \\
\hline Male & 46 & 83.6 \\
\hline Female & 9 & 16.4 \\
\hline \multicolumn{3}{|c|}{ Age, median 61 (range 44-70), years } \\
\hline$\leq 60$ & 27 & 49.1 \\
\hline$>60$ & 28 & 50.9 \\
\hline \multicolumn{3}{|l|}{ Foci } \\
\hline 1 & 50 & 90.9 \\
\hline 2 & 5 & 9.1 \\
\hline \multicolumn{3}{|l|}{ Segment } \\
\hline Upper segment & 11 & 18.3 \\
\hline Middle segment & 38 & 63.3 \\
\hline Lower segment & 11 & 18.3 \\
\hline \multicolumn{3}{|l|}{ T stage } \\
\hline $\mathrm{T} 1$ & 11 & 18.3 \\
\hline T2 & 1 & 1.7 \\
\hline T3 & 47 & 78.3 \\
\hline T4 & 1 & 1.7 \\
\hline \multicolumn{3}{|c|}{ Tumor length, median 5 (range, 2-15) cm } \\
\hline$\leq 5$ & 29 & 48.3 \\
\hline$>5$ & 31 & 51.7 \\
\hline
\end{tabular}

Tokyo, Japan), whose radiopaque part measured $7 \mathrm{~mm}$ in length, with the model name of Olympus HX-610-090S, were placed on tumor superior and inferior boundaries under EUS guidance. Procedures were performed by one single experienced endosonographer. If the endoscope could reach tumor boundaries, one clip was guided and implanted by EUS at each site.

All the patients were checked in a recovery room for one hour after endoscopy and were not given prophylactic antibiotics. They were instructed to contact radiation oncologists if they experienced any postprocedural symptoms. The following adverse events were recorded if they occurred within seven days after endoscopy: esophageal pain, esophageal bleeding, and esophageal perforation according to common terminology criteria for adverse events (CTCAE) version 4.03.

\section{CT simulation and GTV contouring}

The patients underwent a free-breathing contrast-enhanced CT scan for treatment planning on a Siemens Somatom 20 CT simulator (Siemens Medical Solutions, Erlangen, Germany). CT-images were reconstructed in $512 \times 512$ matrices with a voxel size of $0.97 \mathrm{~mm} \times 0.97 \mathrm{~mm} \times 5 \mathrm{~mm}$.

According to the clip markers placed by EUS at the esophageal cancer borders, the reference GTVs were contoured by one experienced radiation oncologist.

Afterward, with the help of the Eclipse 11 treatment planning system (Varian Medical Systems Inc., Palo Alto, CA, U.S.), clip markers on CT were concealed. We created a new structure with the same color as that of the esophageal lumen next to the clip marker and covered the marker to conceal it (Figure 2). Two other radiation oncologists of expertise in esophageal cancer delineated GTVs, which were defined as conventional GTVs, based on esophageal endoscopy and barium radiography findings. Locations of reference and conventional GTVs' superior and inferior borders, as well as GTVs' lengths, were recorded, compared, and analyzed. 


\section{Statistical analysis}

Statistical analysis was performed with the help of SPSS Statistics software, version 19.0 (International Business Machines Corp., Armonk, NY, U.S.). For all tests, the 2 -sided alpha level $<0.05$ was considered significant.

The reference GTVs and conventional GTVs were compared and analyzed. If data conformed to Gaussian distribution, the paired Student's $t$-test was used. Otherwise, the Wilcoxon signed-rank test could be used to determine whether the two GTVs were significantly different. Discrepancies of two GTVs in terms of superior borders, inferior borders, and lengths were calculated. Subgroup analysis was also conducted in different $\mathrm{T}$ stage [early ( $\mathrm{T} 1+$ T2) vs. advanced (T3 + T4)], focus location (upper vs. middle $v s$. lower thoracic segment), and tumor length $(\leq 5 v s .>5 \mathrm{~cm})$ groups.

\section{Results}

\section{Clinical feasibility}

The trial recruited 55 patients with thoracic esophageal squamous cancer (Table 1). Because of multiple primary esophageal tumors, 60 foci were found and marked by titanium clips under EUS. Due to complete esophageal obstruction, EUS could not reach the inferior borders of 9 foci, only placing titanium clips at the corresponding superior borders. The goal to place markers by EUS at the desired locations was achieved in 111 out of 120 borders.

The median interval between EUS-guided implantation of markers and CT simulation was one day (ranging from 0 to 3 days), with the average interval of 1.5 days. All the markers were visible on the planning CT images, except two markers detaching at two foci before simulation. Therefore, 109 metal markers could be seen on simulation CT images.

According to existing metal markers on superior and inferior borders, one experienced physician delineated reference GTVs of all the 60 foci. After that, with efforts by physicists, all the remaining 109 metal markers could be concealed on CT images in the treatment planning system. Two other radiation oncologists with ability in esophageal cancer contoured conventional GTVs of the 60 tumor foci based on endoscopy and barium radiography. Data on border locations and GTVs' lengths were recorded and analyzed.

\section{Adverse events}

According to CTCAE version 4.03, no severe side effects, i.e., grade 3, 4, or 5 adverse events, occurred after implantation of the clip markers. In terms of the main observed adverse events, only seven patients suffered from grade 1 esophageal pain shortly after the placement of metal markers. Nevertheless, the symptoms of mild pain were relieved only in $1-3$ days.

\section{Data analysis}

Before the comparison of two GTVs, recorded data in the whole cohort and subgroups of different $T$ stages, locations, and lengths, were analyzed with the Kolmogorov-Smirnov test and verified as Gaussian distribution. The discrepancy data between reference GTVs and conventional GTVs in every circumstance also conformed to Gaussian distribution, except discrepancy of the inferior border between two GTVs in advanced T stage (T3 + T4) esophageal carcinoma group. As a result, the paired Student's $t$-test was utilized to analyze all the data except that of the inferior border in advanced $\mathrm{T}$ stage cancer, while the latter was analyzed with the Wilcoxon test.

Compared with reference GTVs, the discrepancy of conventional GTVs' superior border was $0.93 \pm 0.82 \mathrm{~cm}$ $(0-3.75 \mathrm{~cm}, \mathrm{P}<0.001)$, while the discrepancy of the inferior border was $0.75 \pm 0.63 \mathrm{~cm}(0-2.75 \mathrm{~cm}, \mathrm{P}<0.001)$. On the contrary, conventional GTVs' length was not significantly different from the reference, with the discrepancy $0.09 \pm 1.32 \mathrm{~cm}(-4.00$ to $4.00 \mathrm{~cm}, \mathrm{P}=0.64)$ (Figure 3).

After we stratified the patients according to distinct local stages, i.e., early $(\mathrm{T} 1+\mathrm{T} 2)$ and advanced $(\mathrm{T} 3+\mathrm{T} 4)$ stage groups, both the discrepancies of conventional GTVs' superior and inferior borders from reference GTVs were significant in either group, but GTVs' length differed insignificantly in any group. If different tumor locations (upper, middle, and lower segments) or tumor lengths ( $\leq 5$ and $>5 \mathrm{~cm}$ ) were taken into consideration, comparable results could be found (Table 2).

\section{Discussion}

Accurate delineation of GTV, which influences the scope of the CTV and PTV significantly, especially in the craniocaudal direction, is one extraordinarily crucial but complicated issue in radiotherapy for esophageal cancer. Although GTV should be delineated on CT images in the overwhelming majority of treatment planning systems, CT alone cannot facilitate accurate contouring of GTV for thoracic esophageal cancer adequately, given the fact that 


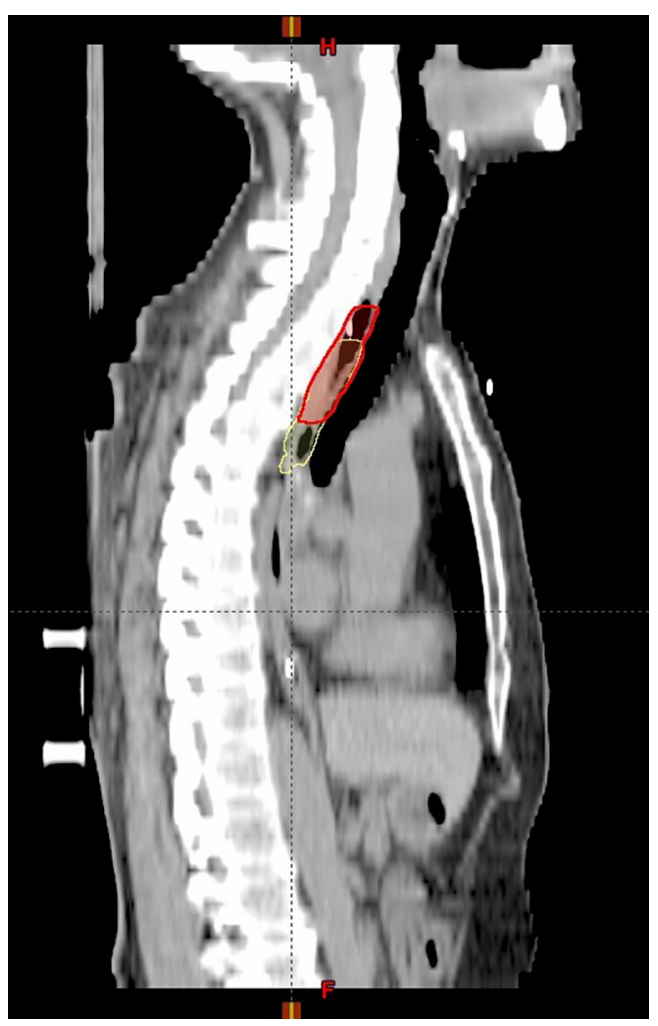

Figure 3 Sagittal CT image of one patient with upper thoracic esophageal cancer, demonstrating difference of conventionally contoured GTV (red) by an experienced radiation oncologist after concealment of titanium clips from reference GTV (yellow), with changes in superior and inferior borders but without change in tumor length. CT, computed tomography; GTV, gross tumor volume. only $32 \%$ consistency between lesions' length measured by CT and measured after cancer surgery was observed (4), probably due to superficial cancer involvement or edema adjacent to tumor. Consequently, in routine practice, we must refer to endoscopy or digestive tract radiography data as well. However, there are also the problems of mismatching because of different patient positions in distinct examinations and esophageal movement during endoscopy or radiography.

MRI (5) and PET (17) have been utilized in some trials to determine the accurate border of esophageal cancer, achieving better results. However, due to the inconsistency of parameter interpretation and higher cost, they were still not the ideal modalities to ease contouring GTVs. In studies on MRI, especially on diffusion-weighted imaging, the best b-value was not determined yet $(5,7)$. Similarly, studies on PET-CT could not reach a consensus about the best paradigm. Some made use of visual interpretation of PET images to determine tumor contour, avoiding geographic misses and reported pathological findings correlated better with PET-based tumor length than with CT scans (17-19), but this method was easily influenced by interobserver variability and display window parameter settings. Yu et al. reported that SUV 2.5 was the best threshold to delineate GTV when comparing with pathological findings after complete esophageal resection. Nevertheless, its conformality index was only 0.52 (11). Uncertainties of parameter values complicated utilization of MRI and PET in determining the scope of esophageal GTV (8-11).

Table 2 Discrepancies of conventional GTVs' superior, inferior borders and length from reference GTVs' in thoracic esophageal cancer of different primary tumor stages, locations, and tumor lengths (centimeter)

\begin{tabular}{|c|c|c|c|c|c|c|}
\hline Esophageal tumor properties & $\begin{array}{c}\text { Discrepancy of superior } \\
\text { border }\end{array}$ & $P$ & $\begin{array}{l}\text { Discrepancy of inferior } \\
\text { border }\end{array}$ & $P$ & $\begin{array}{l}\text { Discrepancy of tumor } \\
\text { length }\end{array}$ & $P$ \\
\hline Early (T1 + T2) & $1.33 \pm 1.20$ & 0.007 & $0.63 \pm 0.43$ & 0.001 & $0.22 \pm 2.07$ & 0.76 \\
\hline Upper segment & $0.68 \pm 0.50$ & 0.001 & $0.81 \pm 0.73$ & 0.022 & $0.91 \pm 0.63$ & 0.38 \\
\hline Middle segment & $0.99 \pm 0.92$ & $<0.001$ & $0.77 \pm 0.63$ & $<0.001$ & $0.18 \pm 1.48$ & 0.51 \\
\hline$\leq 5 \mathrm{~cm}$ & $0.76 \pm 0.61$ & $<0.001$ & $0.72 \pm 0.65$ & $<0.001$ & $0.05 \pm 1.01$ & 0.81 \\
\hline$>5 \mathrm{~cm}$ & $1.07 \pm 0.97$ & $<0.001$ & $0.76 \pm 0.63$ & $<0.001$ & $0.22 \pm 1.56$ & 0.48 \\
\hline
\end{tabular}

GTV, gross tumor volume. 
Besides, the prohibitive cost of these techniques prohibited their routine usage in the clinic.

Marking tumor superior and/ or inferior borders with radiopaque fiducials under endoscope is the most visualized, practical, and economical method to determine GTV scope, especially in terms of longitudinal extent. With the help of EUS, the accuracy of tumor marking could be enhanced because profound tumors could also be detected as well (14). Several studies with small samples of patients with esophageal carcinoma have reported the feasibility and safety of endoscopy/EUS-guided placement of markers in the esophagus (20-22). Fernandez et al. conducted retrospective research with a relatively larger sample size (23). They concluded that the placement of markers with EUS could ease radiation oncologists in many aspects, such as target delineation and image guidance during delivery of radiotherapy with cone-beam CT.

To the best of our knowledge, this research is the first registered prospective trial with adequate size to evaluate the role of clip markers placed by EUS in GTV delineation of thoracic squamous esophageal cancer. This prospective study revealed the significant difference between GTVs delineated with the conventional method and the approach eased by EUS-placed titanium clips, regardless of T stage, location, or tumor length. Besides, no severe adverse events, e.g., intolerable esophageal pain, bleeding or perforation, occurred during or after clip placement. The results illuminated the critical role of EUS in precise treatment for thoracic esophageal squamous carcinoma.

Even in locally advanced tumors, i.e., T3 or T4, which had relatively recognizable borders, the difference between the two methods could reach as high as 0.84 and $0.78 \mathrm{~cm}$ in superior and inferior borders, respectively, more than two slices with a slice thickness of $3 \mathrm{~mm}$. This phenomenon might be explained by edema or superficial involvement at tumor borders, which could lead to overestimation or underestimation of tumor extents by CT. Consequently, we recommend it is critical to place clip markers with EUS before radiotherapy simulation for patients with thoracic esophageal squamous carcinoma of all stages, not only in locally early tumors.

Even when novel techniques were taken into consideration, the role of the clip marker remained robust. Thomas et al. reported that although PET-CT was highly relevant for staging purposes, CT with clipping of tumor borders remained the best method for GTV delineation (6). Compared with CT images with metal markers, no PETbased algorithm performed better. That study was a head- to-head comparison trial, but, unlike ours, it utilized asynchronous images to compare GTVs marked by endoscopy-guided clip markers and PET-CT, requiring complex image registration and hence introducing additional error, complicating interpretation of the results. On the contrary, we concealed the titanium clips on CT images, making only one CT scan enough, avoiding the registration error and exposure of patients to further irradiation.

Besides, Cuellar et al. reported that PET-CT was not useful in the evaluation of early stage esophageal adenocarcinoma (24). Consequently, the authors believed that FDG-PET/CT should not be routinely performed for early-stage esophageal cancer. That meant clipping by EUS was more useful than PET in the context of early stage esophageal cancer.

One interesting finding of our research was that although oncologists contoured significantly different GTVs with the two methods, tumor lengths were the same. The length posed a question for some previous studies that utilized tumor length as the object of comparison among different methods, such as PET-CT (25), MRI (7), and pathology. We suppose the same gross tumor length should not necessarily equal to the same GTV. Tumor length may not be the touchstone of better GTV delineation approaches in future trials.

Although we defined GTVs delineated with the help of clip markers as a reference, it must be pointed out that the gold standard determining esophageal carcinomas' GTV borders is pathological finding. Nevertheless, no data has been publicized to compare border locations between EUS data and pathological specimens at present. Our future work will place clip markers by EUS on esophageal tumor borders before resection during the operation and contrast the locations of clip markers with pathological tumor borders, to investigate whether EUS could be the surrogate of pathology as the gold standard.

The disadvantages of EUS-based clipping included occasional inability to pass through esophageal lumen due to cancer obstruction and clip detachment. For cancer obstruction, our solution was to take tumor length data of the barium radiograph into consideration. We assumed the tumor length measured by barium radiograph as correct, so the position of inferior border could be determined by the clip marked at the superior border. Clip detachments were rare, accounting for only $1.8 \%$ (2 out of 111 ) of all placements in our trial. The rarity might be attributed to the interval between placement of clip markers and CT 
simulation. The average interval was 1.5 days in our trial. Our solution for clip detachment was to shorten the interval to less than one day, i.e., undertaking CT simulation shortly after EUS.

In summary, we suggest utilization of EUS-placed clips in delineation of GTVs for patients with thoracic esophageal cancer.

\section{Conclusions}

This study confirmed that EUS-placed titanium clips could facilitate accurate contouring of GTVs in thoracic esophageal cancer in different $\mathrm{T}$ stages and locations but could not affect GTV length. Consequently, we recommend routine use of EUS-placed titanium clips in the practice of radiotherapy for thoracic esophageal cancer. Compared to methods using barium radiography, MRI, or PET, it could ease delineating esophageal GTVs precisely and economically.

\section{Acknowledgments}

Funding: General Project of National Nature Foundation (China), Grant No. 81872462. Clinical Trial Supporting Foundation of Tianjin Medical University Cancer Institute \& Hospital (Grant No. C1707).

\section{Footnote}

Reporting Checklist: The authors have completed the STROBE reporting checklist. Available at http://dx.doi. org/10.21037/atm-20-4030

Data Sharing Statement: Available at http://dx.doi. org/10.21037/atm-20-4030

Conflicts of Interest: All authors have completed the ICMJE uniform disclosure form (available at http://dx.doi. org/10.21037/atm-20-4030). The authors have no conflicts of interest to declare.

Ethical Statement: The authors are accountable for all aspects of the work in ensuring that questions related to the accuracy or integrity of any part of the work are appropriately investigated and resolved. All procedures performed in this study involving human participants were in accordance with the Declaration of Helsinki (as revised in 2013). The study was approved by institutional ethics committee of Tianjin Medical University Cancer Institute and Hospital (No. E2016103A) and informed consent was taken from all the patients.

Open Access Statement: This is an Open Access article distributed in accordance with the Creative Commons Attribution-NonCommercial-NoDerivs 4.0 International License (CC BY-NC-ND 4.0), which permits the noncommercial replication and distribution of the article with the strict proviso that no changes or edits are made and the original work is properly cited (including links to both the formal publication through the relevant DOI and the license). See: https://creativecommons.org/licenses/by-nc-nd/4.0/.

\section{References}

1. Bray F, Ferlay J, Soerjomataram I, et al. Global cancer statistics 2018: GLOBOCAN estimates of incidence and mortality worldwide for 36 cancers in 185 countries. CA Cancer J Clin 2018;68:394-424.

2. Minsky BD, Pajak TF, Ginsberg RJ, et al. INT 0123 (Radiation Therapy Oncology Group 94-05) phase III trial of combined-modality therapy for esophageal cancer: high-dose versus standard-dose radiation therapy. J Clin Oncol 2002;20:1167-74.

3. Deng W, Lin SH. Advances in radiotherapy for esophageal cancer. Ann Transl Med 2018;6:79.

4. Drudi FM, Trippa F, Cascone F, et al. Esophagogram and CT vs endoscopic and surgical specimens in the diagnosis of esophageal carcinoma. Radiol Med 2002;103:344-52.

5. Hou DL, Shi GF, Gao XS, et al. Improved longitudinal length accuracy of gross tumor volume delineation with diffusion weighted magnetic resonance imaging for esophageal squamous cell carcinoma. Radiat Oncol 2013;8:169.

6. Thomas L, Lapa C, Bundschuh RA, et al. Tumour delineation in oesophageal cancer - A prospective study of delineation in PET and CT with and without endoscopically placed clip markers. Radiother Oncol 2015;116:269-75.

7. Liu G, Yang Z, Li T, et al. Optimization of b-values in diffusion-weighted imaging for esophageal cancer: Measuring the longitudinal length of gross tumor volume and evaluating chemoradiotherapeutic efficacy. J Cancer Res Ther 2017;13:748-55.

8. Hong TS, Killoran JH, Mamede M, et al. Impact of manual and automated interpretation of fused PET/CT data on esophageal target definitions in radiation planning. 
Int J Radiat Oncol Biol Phys 2008;72:1612-8.

9. Dong X, Wu P, Sun X, et al. Intra-tumour 18F-FDG uptake heterogeneity decreases the reliability on target volume definition with positron emission tomography/ computed tomography imaging. J Med Imaging Radiat Oncol 2015;59:338-45.

10. Guo Y, Li J, Zhang P, et al. Comparative evaluation of target volumes defined by deformable and rigid registration of diagnostic PET/CT to planning CT in primary esophageal cancer. Medicine 2017;96:e5528.

11. Yu W, Fu XL, Zhang YJ, et al. GTV spatial conformity between different delineation methods by 18FDG PET/ CT and pathology in esophageal cancer. Radiother Oncol 2009;93:441-6.

12. Thosani N, Singh H, Kapadia A, et al. Diagnostic accuracy of EUS in differentiating mucosal versus submucosal invasion of superficial esophageal cancers: a systematic review and meta-analysis. Gastrointest Endosc 2012;75:242-53.

13. Riepl M, Pietsch A, Klautke G, et al. Endoscopic pretherapeutic clipping for gastrointestinal tumors. A method for exact definition of the target volume. Strahlenther Onkol 2000;176:517-23.

14. Kelly S, Harris KM, Berry E, et al. A systematic review of the staging performance of endoscopic ultrasound in gastro-oesophageal carcinoma. Gut 2001;49:534-9.

15. He LJ, Shan HB, Luo GY, et al. Endoscopic ultrasonography for staging of T1a and T1b esophageal squamous cell carcinoma. World J Gastroenterol 2014;20:1340-7.

16. Yang J, Luo GY, Liang RB, et al. Efficacy of Endoscopic Ultrasonography for Determining Clinical T Category for Esophageal Squamous Cell Carcinoma: Data From 1434 Surgical Cases. Ann Surg Oncol 2018;25:2075-82.

17. Leong T, Everitt C, Yuen K, et al. A prospective study to evaluate the impact of FDG-PET on CT-based

Cite this article as: Guan Y, Wang J, Cao F, Chen X, Wang Y, Jiang S, Zhang D, Zhang W, Guo Z, Wang P, Pang Q. Role of clip markers placed by endoscopic ultrasonography in contouring gross tumor volume for thoracic esophageal squamous cell carcinoma: one prospective study. Ann Transl Med 2020;8(18):1144. doi: 10.21037/atm-20-4030 radiotherapy treatment planning for oesophageal cancer. Radiother Oncol 2006;78:254-61.

18. Konski A, Doss M, Milestone B, et al. The integration of 18-fluoro-deoxy-glucose positron emission tomography and endoscopic ultrasound in the treatment-planning process for esophageal carcinoma. Int J Radiat Oncol Biol Phys 2005;61:1123-8.

19. Gondi V, Bradley K, Mehta M, et al. Impact of hybrid fluorodeoxyglucose positron-emission tomography/ computed tomography on radiotherapy planning in esophageal and non-small-cell lung cancer. Int J Radiat Oncol Biol Phys 2007;67:187-95.

20. Pfau PR, Pham H, Ellis R, et al. A novel use of endoscopic clips in the treatment planning for radiation therapy (XRT) of esophageal cancer. J Clin Gastroenterol 2005;39:372-5.

21. Pishvaian AC, Collins B, Gagnon G, et al. EUS-guided fiducial placement for CyberKnife radiotherapy of mediastinal and abdominal malignancies. Gastrointest Endosc 2006;64:412-7.

22. DiMaio CJ, Nagula S, Goodman KA, et al. EUS-guided fiducial placement for image-guided radiation therapy in GI malignancies by using a 22-gauge needle (with videos). Gastrointest Endosc 2010;71:1204-10.

23. Fernandez DC, Hoffe SE, Barthel JS, et al. Stability of endoscopic ultrasound-guided fiducial marker placement for esophageal cancer target delineation and image-guided radiation therapy. Pract Radiat Oncol 2013;3:32-9.

24. Cuellar SL, Carter BW, Macapinlac HA, et al. Clinical staging of patients with early esophageal adenocarcinoma: does FDG-PET/CT have a role? J Thorac Oncol 2014;9:1202-6.

25. Jeganathan R, McGuigan J, Campbell F, et al. Does preoperative estimation of oesophageal tumour metabolic length using 18F-fluorodeoxyglucose PET/CT images compare with surgical pathology length? Eur J Nucl Med Mol Imaging 2011;38:656-62. 